\title{
Contributi italiani alla nascita della Petrografia ${ }^{\dagger}$
}

\author{
R. Cirrincione[1]* \& R. Cristofolini[2] \\ [1] Dipartimento di Scienze Biologiche, Geologiche, Ambientali, Sez. Sci. Terra, Università di Catania \\ [2] già Professore di Vulcanologia, Università di Catania
}

\begin{abstract}
Riassunto
Verso la fine del XVIII e nei primi decenni del XIX secolo alcuni esperti italiani nella prospezione e sfruttamento minerario ed interessati nelle Scienze della Terra, G. Arduino, G. Marzari Pencati, G. Brocchi, and S. Breislak, hanno correttamente riconosciuto in base ad osservazioni sul campo la natura che oggi definiremmo magmatica di corpi sia intrusivi che vulcanici all'interno di sequenze sedimentarie, in contrasto con quanto previsto dalle teorie nettunistiche di Werner, allora dominanti. Ma anche celebrati studiosi di Storia Naturale, come L. Spallanzani e G. Gioeni hanno dato un importante contributo alle ricerche sulle rocce, ed al termine del periodo è da ricordare anche C. Gemmellaro, successore di Gioeni. La Petrografia si stava allora sviluppando, nella cornice della Storia Naturale, come disciplina autonoma dalla Mineralogia e dalla Geologia quando fu possibile classificare le rocce, già suddivise da Werner in gruppi fondati su criteri sistematici embrionalmente petrografici, sulla base dei primi fondamentali lavori di Cordier (1816), von Leonhard (1823) e Brongniart (1827), che hanno introdotto una tassonomia delle rocce, ancora affetta da inconsistenze ed ingenuità, in base alla loro composizione ed origine.
\end{abstract}

Parole chiave: Petrografia, Storia della Scienza, secoli XVIII e XIX, Italia

\section{Summary}

\section{Italian contributions to the birth of Petrography}

At the end of the $18^{\text {th }}$ and in the first decades of the $19^{\text {th }}$ century G. Arduino, G. Marzari Pencati, G. Brocchi, and S. Breislak, italian experts in ore prospecting and exploitation, and interested in Earth sciences, correctly recognized the igneous nature of either intrusive or volcanic bodies within sedimentary sequences on the grounds of field data, in contrast to Werner's neptunistic ideas. But also renown scholars in the field of Natural History, as L. Spallanzani and G. Gioeni gave relevant contributions to the study of rocks, and at the end of this stage C. Gemmellaro is to be cited, as a successor of Gioeni. Petrography started to develop when rocks, already subdivided by Werner (1774) into groups meeting some criteria of petrographical taxonomy. It reached the stage, as an autonomous discipline from Mineralogy and Geology, within the frame of Natural History, when rocks could be classified on the grounds of the basic works by Cordier (1816), von Leonhard (1823), and Brongniart (1827), who introduced a taxonomic basis, although still affected by inconsistencies and naiveties, related to rocks on the grounds of their compositions and origin.

Key words: Petrography, Science history, $18^{\text {th }}$ and $19^{\text {th }}$ centuries, Italy

\footnotetext{
${ }^{\dagger}$ Nota presentata nell’Adunanza pubblica del 5 maggio 2017
}

*e-mail: r.cirrincione@unicit.it 


\section{Introduzione}

Alla fine del XVIII secolo e nei primi decenni del XIX studiosi formati e residenti nella penisola italiana hanno dato significativi contributi allo sviluppo delle conoscenze nell'ambito di quelle che oggi si definiscono come Scienze della Terra. Tra questi in particolare alcuni esperti italiani in prospezione e coltivazione mineraria, G. Arduino, G. Marzari Pencati, G. Brocchi, and S. Breislak, con notevole predisposizione all'interpretazione di quanto osservato nelle loro indagini, hanno correttamente riconosciuto la natura magmatica di corpi rocciosi sia vulcanici che intrusivi, riscontrati all'interno di successioni sedimentarie, in base alle loro caratteristiche riscontrate in campagna. Ciò era in accordo con quanto avrebbe riconosciuto nelle isole britanniche Hutton (1788) e contrastante con le ipotesi di lavoro elaborate da Werner (1774), riprese da molti studiosi del tempo e rielaborate nel modello nettunistico; in base a questo, dominante per molti anni, si riteneva che le rocce affioranti alla superficie terrestre avessero avuto origine per precipitazione da soluzioni in un oceano che in origine ricopriva tutta la la Terra e che andava progressivamente ritirandosi. Oltre a questi ricercatori agli inizi dello stesso periodo anche riconosciuti studiosi di Storia Naturale, come Lazzaro. Spallanzani e Giuseppe Gioeni, che avevano rivolto i loro interessi allo studio di vulcani attivi come il Vesuvio e l'Etna, hanno contribuito in modo significativo allo studio dei materiali rocciosi, mentre alla fine di esso si deve ricordare Carlo Gemmellaro, che fu un successore di Gioeni.

Era il momento in cui l'embrione della petrografia stava crescendo affermandosi come disciplina autonoma dalle sue "madri", la Mineralogia e la Geologia, a loro volta in rapido sviluppo, entro la cornice della Storia Naturale, in un momento storico in cui si ampliava il quadro delle conoscenze in campo fisico e chimico con importanti apporti nelle tecniche sperimentali e analitiche. Dopo che Werner (1774) aveva già suddiviso le rocce in base alla loro natura in gruppi rispondenti a criteri in qualche modo petrografici, nei primi decenni del XIX secolo, i lavori di Cordier (1816), von Leonhard (1823), and Brongniart (1827) hanno introdotto nell'ambito delle ricerche basi originali, benché ancora affette da notevoli problemi di inconsistenza ed ingenuità, per la tassonomia delle rocce in relazione a dati sulla loro composizione intesi ad interpretarne l'origine. Si stava così completando una gestazione millenaria, sostenuta dall'accrescimento delle conoscenze scientifiche e dal conseguente evolversi delle tecniche e dei metodi di ricerca iniziata nei secoli immediatamente precedenti. Come primo tentativo moderno di classificazione delle rocce si può ricordare quello introdotto da Linneo nel capitolo Petrae del Regnum lapideum nella $10^{\mathrm{a}}$ edizione del Systema Naturae (1758): questo schema, basato su classi, generi e specie, benché inadatto alla classificazione di oggetti del mondo inorganico, fu usato all'inizio da numerosi ricercatori (p.es. Haüy, 1822).

Prima dell'introduzione del microscopio polarizzante da parte di Sorby (1858), solamente le osservazioni si campagna, accompagnate dall'esame di campioni a scala mesoscopica e da dati chimici approssimativi, costituivano la base fondamentale dello studio delle rocce e dei loro componenti, fino a quando P.-L. Cordier (1816) non ha introdotto criteri classificativi basati sul riconoscimento dei loro componenti minerali, identificati mediante l'esame microscopico di campioni polverizzati. A quel tempo anche la classificazione dei minerali era assai elementare, essendo basata essenzialmente sull'esame qualitativo del loro aspetto. Non si potevano allora definire dettagli sulle strutture delle rocce e riconoscerne le associazioni minerali caratteristiche.

In ogni caso già da decenni lo studio dei minerali e quindi delle rocce si stava sviluppando in conseguenza della crescente disponibilità di dati prodotti da fisici e matematici (Kepler, 1611; Stenone, 1669; Romé de l'Isle, 1783, 1784; Haüy, 1784, 1801) sui caratteri cristallografici e le proprietà fisiche delle sostanze solide, nonché dai chimici su atomi, elementi, loro composti e sulle modalità e leggi di reazione (Boyle, Lavoisier, Proust, Dalton, Davy, Gay Lussac, Berzelius,...). D’altro canto i "geologi" (prospettori minerari, studiosi di Storia Naturale) erano sempre più coinvolti in attività di ricerca sul campo e/o nel raccogliere e classificare collezioni di rocce da esporre in un crescente numero di "gabinetti" o musei. 


\section{Il contributo di ricercatori italiani}

$\mathrm{Nel}$ quadro sopra tratteggiato i sopra citati ricercatori italiani, che vivevano in una terra ancora definita "un'espressione geografica" da Metternich nel 1847, ancora suddivisa in molti stati e fin dal Medio Evo solamente conosciuta come "il bel paese che il mar circonda e l'Alpe" (Petrarca, Canzon.) e "serva Italia di dolore ostello, nave senza nocchiero in gran tempesta" (Dante, Purg. VI), erano impegnati anche in ricerche sulle rocce e sulle loro caratteristiche. Erano appartenenti alla nobiltà o alla classe borghese, talora religiosi, che contribuito in modo rilevante alla nascita e sviluppo della petrografia, nel momento storico particolarmente turbolento dal punto di vista sociale politico che va dagli ultimi decenni del XVIII secolo, attraverso la Rivoluzione Francese ed il periodo Napoleonico, fino alle Restaurazione successiva al 1814 (v. Tav. 1 a-f).

In questo quadro appare rilevante notare che gli studiosi sopra citati hanno avuto importanti rapporti diretti ed epistolari con i maggiori cultori europei contemporanei delle discipline di loro interesse nell'ambito della Storia naturale, e che le loro opere hanno avuto un'estesa circolazione e positivi riconoscimenti nel mondo scientifico. In particolare, anche a motivo dei rivolgimenti socio-politici tra la fine del 1700 ed i primi decenni del 1800, essi hanno avuto modo di trascorrere prolungati soggiorni presso alcune delle principali scuole in cui si stavano sviluppando i nuovi concetti nel campo delle scienze della Terra. Inoltre risulta significativo che tra questi abbiano dato un importante contributo allo sviluppo in Italia di conoscenze minero-petrografiche e geologiche, non solo appartenenti al mondo "accademico", ma anche esperti in attività legate alla ricerca ed allo sfruttamento di giacimenti minerari, nonché allo sviluppo di tecniche ed attrezzature per la lavorazione dei materiali estratti, che si erano andate accrescendo ed affinando empiricamente nel corso dei millenni, portando quindi alla produzione di oggetti metallici, laterizi, ceramiche e vetri.

2a Giovanni Arduino (1714 - 1795; Figg. 1-2), è noto particolarmente come fondatore, già riconosciuto da Gemmellaro (1862), di una stratigrafia fondata sul riconoscimento di un'ordinata successione di litotipi nel corso delle sue indagini nell'Italia nord-orientale $(1760,1774)$, rivolte prevalentemente alla prospezione mineraria ed a rilevamenti in campagna ad esse correlati nella Repubblica di Venezia e nel Granducato di Toscana (Tav. 1 a). Come risultato delle sue osservazioni egli ha riconosciuto che limitati livelli affioranti nelle zone prossime a Verona e Vicenza intercalati in sequenze sedimentarie da lui definite come terziarie e secondarie erano prodotti da episodi vulcanici, dovuti a "effetti di fuochi sotterranei molto antichi". Ha così contribuito a dare una soluzione anche alla controversia se l'origine di rocce definite come basalti fosse per "via umida" (deposizione da soluzioni) o per "azione del fuoco" (solidificazione di fusi), oltre che a superare il modello nettunista.

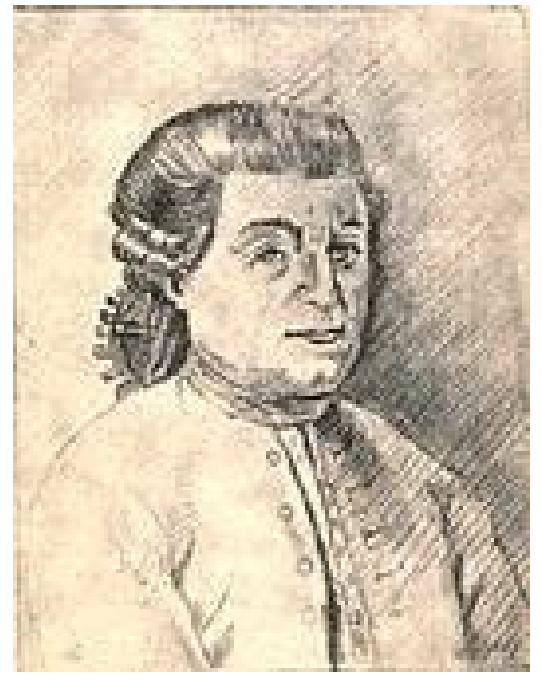

Fig. 1 - Giovanni Arduino

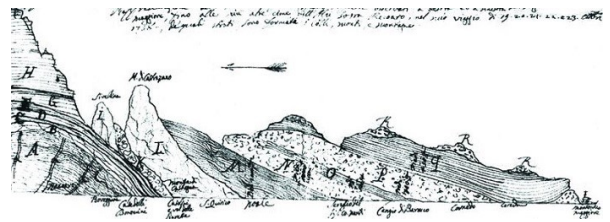

Fig. 2 - Una sezione geologica di Arduino (1760) 
Successivamente, nel 1782 Arduino ricorda i vari passi delle sue indagini su diversi materiali sopra citati correttamente da lui interpretati come vulcanici, attribuendosi il merito di essere stato il primo ad averne riconosciuto l'origine, e poi nel 1792 riporta il ritrovamento di altri livelli vulcanici da lui ritrovati nell'Italia nord-orientale, allora parte della Repubblica Veneta o sotto il dominio dell' Austria (Vaccai, 2013 e riferimenti).

1760, Due lettere del Sig.Giovanni Arduino sopra varie sue osservazioni naturali, «Nuova raccolta di opuscoli scientifici e filologici», 6, pp. XCIX-CLXXX.

1774, Saggio fisico-mineralogico di lythogonia e orognosia, «Atti dell'Accademia delle scienze di Siena detta de' Fisiocritici», 1774, 5, pp. 228-300.

1782, Memoria epistolare sopra varie produzioni vulcaniche, minerali e fossili, Venezia.

1792, Circa gl'indizi d'antichissimi vulcani nelle montagne e Alpi Vicentine, Veronesi, e Trentine, «Memorie di matematica e fisica della Società italiana», 6, pp.102-105.

2b Lazzaro Spallanzani (1729 - 1799; Fig. 3), nato nel Ducato di Modena (Tav. 1 a), studente di Giurisprudenza all'Università di Bologna, si laureò in Filosofia (1755) e fu ordinato nell'Ordine dei Gesuiti (1763). Nel frattempo si stava sviluppando in lui l'interesse per la Storia Naturale, particolarmente rivolto a ricerche sugli esseri viventi. A seguito dei primi rilevanti risultati delle sue ricerche, nel 1769 fu nominato dall'Imperatrice Maria Teresa professore di Storia Naturale nell'Università di Pavia, allora parte del Ducato di Milano, sotto dominio austriaco, dove organizzò un ricco museo contenente anche collezioni di minerali e rocce ed ebbe a disposizione laboratori attrezzati per condurre le sue attività di ricerca (Mazzarello, 2013).

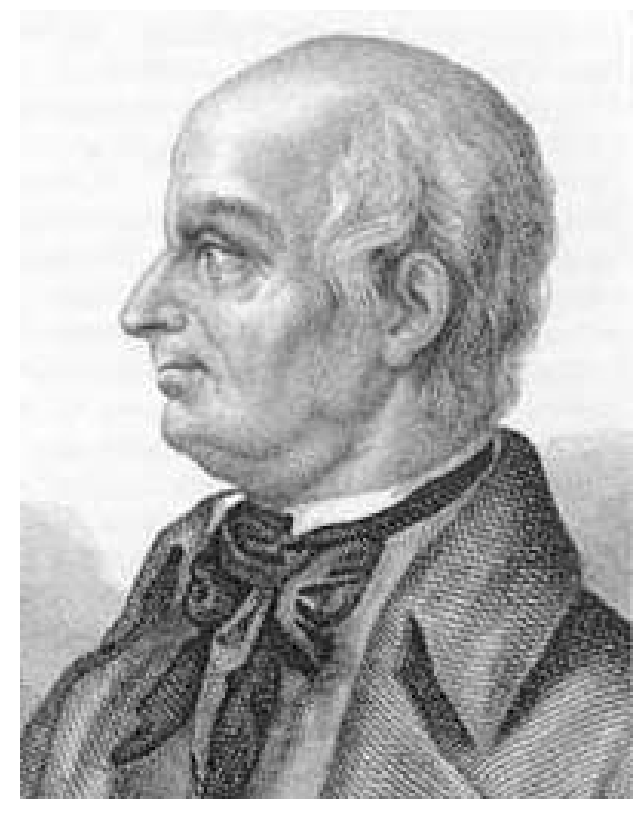

Fig. 3 - Lazzaro Spallanzani

Benché universalmente noto principalmente come precursore nell' ambito delle ricerche a carattere biologico (Mazzarello, 2013 e riferimenti), Spallanzani rivolse il suo interesse anche su tematiche nel campo delle Scienze della Terra: in questo campo il suo contributo di maggiore rilevanza riguardante le caratteristiche di minerali e rocce, particolarmente di origine vulcanica, e sulla loro origine appare in varie pardi dell'opera Viaggi alle Due Sicilie e in alcune parti dell'Appennino (6 voll, 1792-1797), un dettagliato resoconto sulle osservazioni compiute durante un viaggio effettuato nel 1788 nell'Italia meridionale (Regno di Napoli e di Sicilia: Tav. 1 a), in cui riferisce anche su diversi aspetti presentati dalle regioni vulcaniche attive che aveva visitato 
(Vesuvio, Isole Eolie, Etna), con particolare riferimento agli aspetti della loro attività, ai litotipi e materiali emessi e sulle loro proprietà fisiche e caratteri chimici, rilevabili mediante le tecniche disponibili al suo tempo. In questo quadro appaiono di particolare rilievo gli esperimenti di fusione eseguiti su minerali e lave dell'Etna, in quanto prodromici alla successiva petrologia sperimentale: infatti riferisce di avere riscontrato che il feldspato (plagioclasio) fonde alla temperatura raggiunta nella fornace di cui disponeva, il sorlo (augite) fonde solo se "incorporato nella sua lava" e che in ogni caso il prodotto di fusione raffreddato è un vetro omogeneo. In altra parte di questo lavoro egli espone anche dati sulla chimica e mineralogia di fanghi argillosi emessi da alcune "salse" (vulcani di fango) nell'Appennino nei pressi di Modena.

1792 - 1797, Viaggi alle Due Sicilie e in alcune parti dell'Appennino (6 voll.).

2c Giuseppe Gioeni (1743 - 1822; Fig. 4), noto Cavaliere appartenente alla famiglia dei Duchi di Angiò, quando nel 1780 donò una sua collezione di rocce e minerali Etnei, oltre che di conchiglie e fossili siciliani all'Università di Catania fu nominato professore di Storia Naturale (e Botanica fino al 1788) nella Classe (Facoltà) di Medicina della stessa (Cristofolini, 2016 e riferimenti), da parte del Re Ferdinando, IV di Napoli e III di Sicilia (Tav. 1a). L'interesse di Gioeni per le scienze della Terra, nell'ambito dello studio della Natura nacque dapprima attraverso contatti con Giuseppe Recupero (1720-1778) e l'amicizia con Ignazio Paternò Castello, principe di Biscari (1719-1786), ed ebbe un notevole sviluppo dopo che aveva letto il lavoro Campi Phlegraei (1776) di sir W. Hamilton, plenipotenziario britannico alla corte borbonica di Napoli, con cui ebbe rapporti di amicizia. Nel 1781 incontrò a Catania anche Déodat de Dolomieu, con il quale mantenne un continuo rapporto epistolare (Bruccheri, 2001).

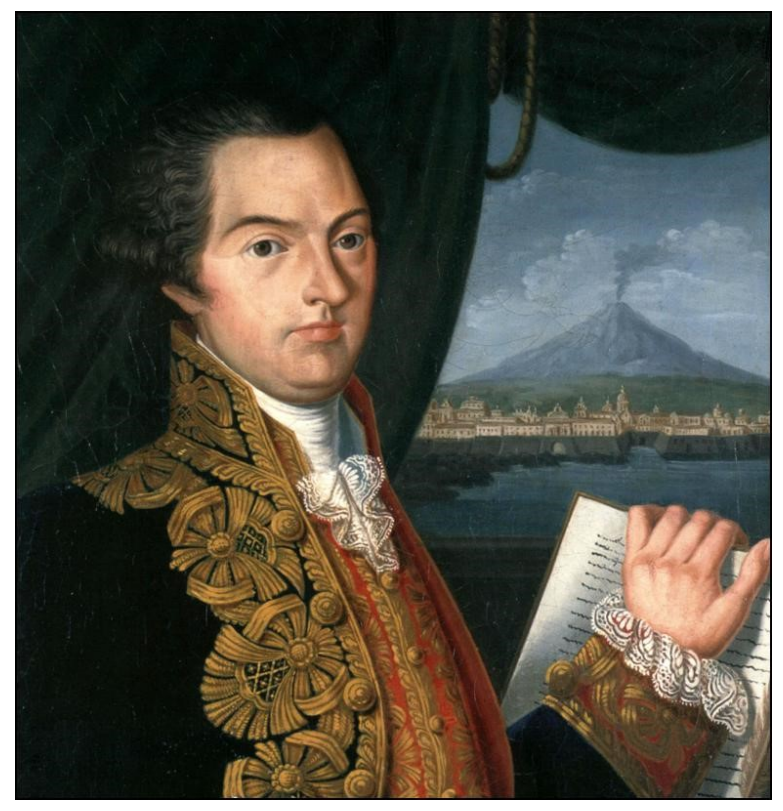

Fig. 4 - Giuseppe Gioeni 

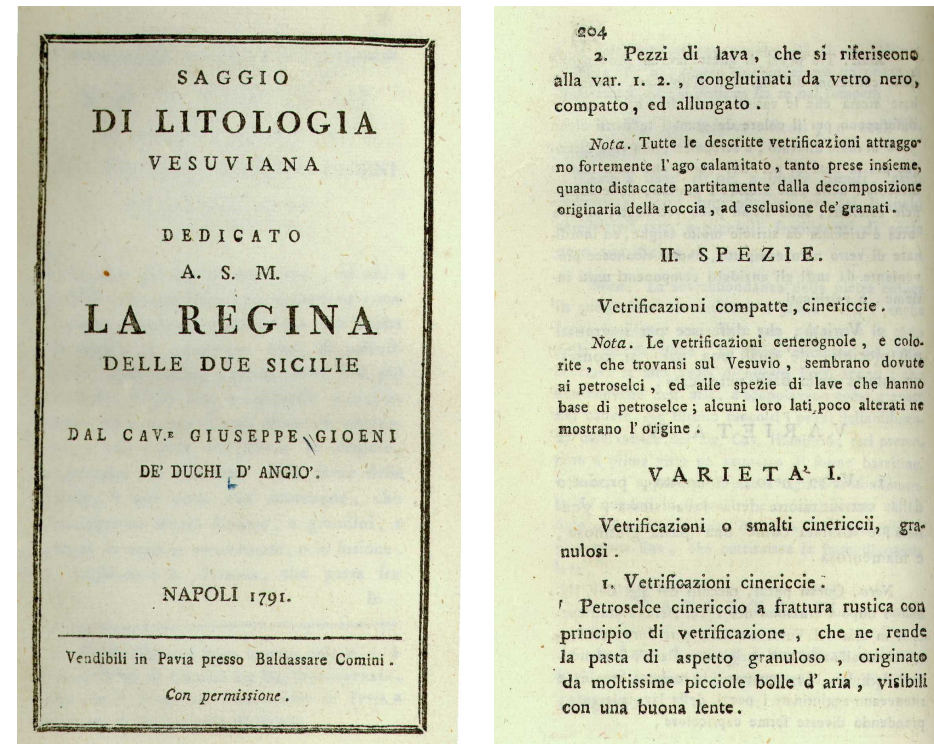

Fig. 5 - Frontespizio e una pagina del Saggio di Litologia Vesuviana

Dopo una relazione sull'eruzione Etnea del 1787, pubblicò a Napoli nel 1791 il suo lavoro certamente di maggiore rilevanza, il Saggio di litologia vesuviana, che può essere considerato uno dei primi lavori a carattere petrografico regionale, in cui particolari varietà di prodotti del Vesuvio sono state classificate secondo una schema Linneano, Nell'introduzione Gioeni rivendica la priorità dei dati sperimentali e di osservazione rispetto alle teorie basate su principi generali non verificati. Il catalogo che segue è compilato secondo quanto premesso suddividendo i Sassi primordiali da quelli vulcanizzati, trasformati cioè dall'azione del fuoco, a loro volta suddivisi in Classi, Generi e Specie (a loro volta suddivise in Varietà, Fig. 5), al fine di esporre i caratteri più significativi per interpretarne l'origine ed i rapporti reciproci.

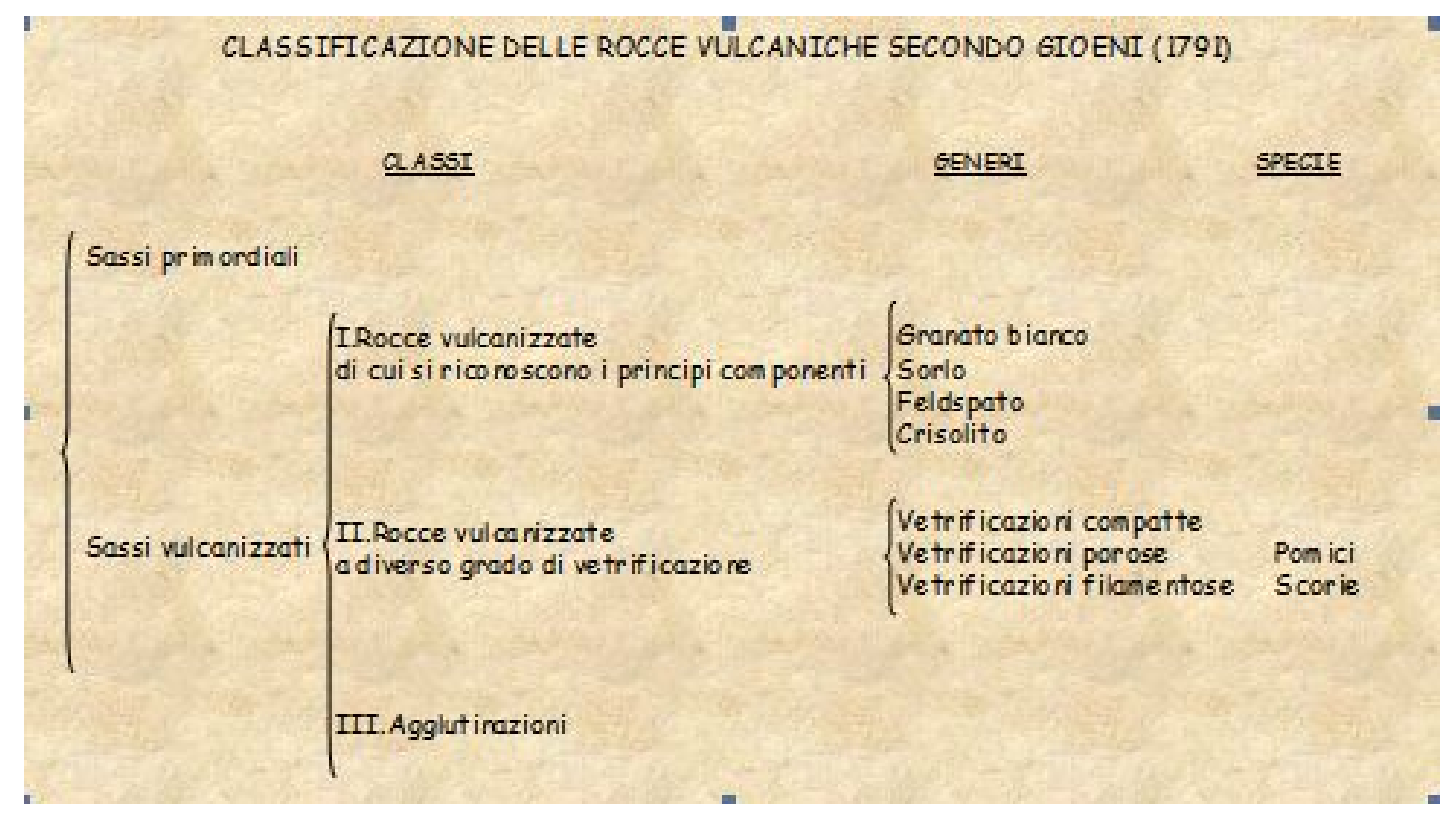

Successivamente proseguì a collezionare materiali per il suo museo personale ed a interessarsi di prodotti dell'Etna, senza tuttavia pubblicare significativi risultati del suo lavoro, fino alla fine del XVIII secolo. Allora carico di debiti fino a 12.000 once, per l'acquisto di strumenti, libri e collezioni di oggetti naturali, non essendo riuscito a vendere queste ultime al Re, iniziò a peregrinare per l'Italia, sottoposta in quegli anni a frequenti cambiamenti politici, e fu rimosso nel 1804 dalla sua cattedra, tenuta poi da Girolamo Recupero. Tornato in Sicilia nel 1811, fu imprigionato 
per debiti per quasi tre anni, al termine dei quali rientrò da privato nella sua dimora catanese, dove riordinò per quanto possibile le sue collezioni si occupò attivamente, senza riuscirvi, di istituire un'accademia che riunisse i migliori studiosi locali di Storia Naturale.

1787, Relazione della eruzione dell'Etna nel mese di luglio 1787, Catania.

1791, Saggio di litologia vesuviana, Napoli.

2d Giambattista (Giovanni Battista) Brocchi (1772 - 1826; Figg. 6-7). Nato a Bassano (nella Repubblica di Venezia, Tav. 1 a) dapprima frequentò corsi umanistici e teologici presso l'Università di Padova, ma fu ben presto attratto dallo studio della Storia naturale. Nel 1801 dopo l'instaurazione della napoleonica Repubblica Cisaplpina (poi Repubblica Italiana, 1802 (Tav. 1 b) e Regno d'Italia, 1805, (Tav. 1 c), fu Insegnante presso la scuola superiore di Brescia, della quale riordinò, arricchì e studiò approfonditamente le collezioni di minerali e rocce della Lombardia. A motivo delle sue attività rivolte anche allo studio di aspetti geologici e mineralogici di giacimenti minerari e del loro sfruttamento, nel 1809 fu nominato Ispettore del Consiglio delle Miniere del Regno d'Italia. Dopo la sconfitta di Napoleone nel 1814 e la restaurazione di molti degli stati preesistenti (Tav. $1 \mathrm{~d}$ ) egli perse l'impiego ed iniziò a viaggiare in gran parte della penisola Italiana, studiandone la geologia e litologia (Giacomini, 1972 e riferimenti) pubblicando i risultati delle sue attente osservazioni.

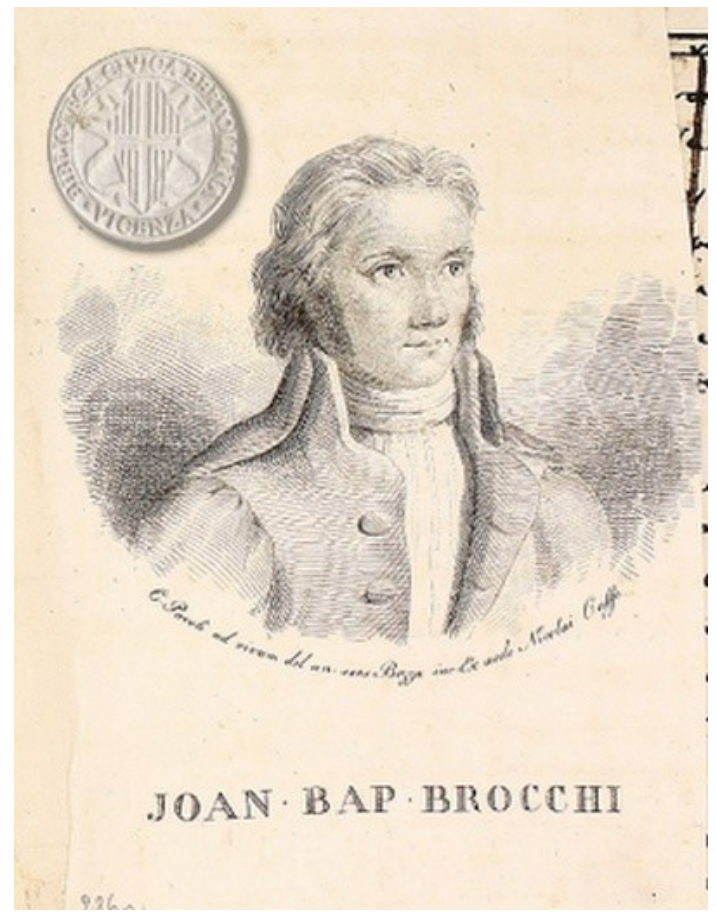

Fig. 6 - Giambattista Brocchi

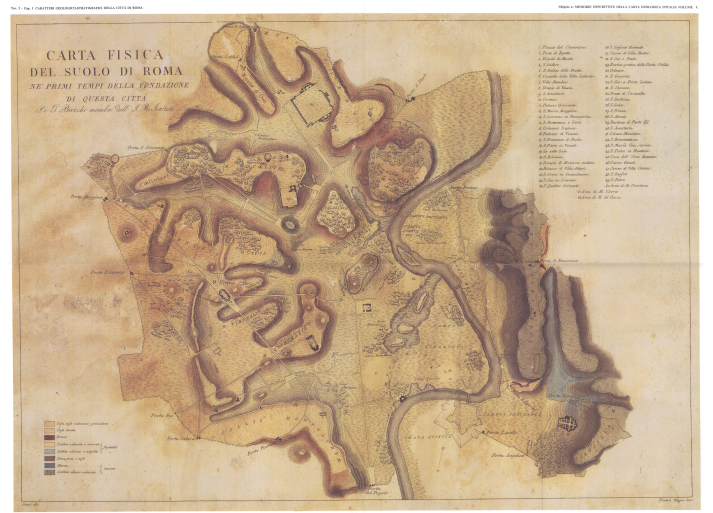

Fig. 7 - Carta geognostica di Roma (1820) di G.B. Brocchi

Benché il suo più importante contributo, pubblicato nel 1814 sia di interesse stratigrafico, riportando importanti dettagli sulla struttura della catena Appenninica e le prove che i fossili giacenti negli strati più profondi appartengono a specie estinte e vengono gradualmente sostituiti da altri, già nel 1811, Brocchi scrisse un importante lavoro sui minerali riscontrati al contatto di una massa rocciosa, interpretata come magmatica, con rocce sedimentarie calcaree, concordando con quanto riscontrato da Arduino (1792). Nel 1817 vide la luce un suo catalogo di rocce inteso come guida alle ricerche geognostiche in Italia, e nel 1820 interpretò correttamente i prodotti affioranti nell'area di Roma come dovuti ad attività vulcanica esplosiva di vulcani inattivi situati nei Colli Albani o Cimini. Ha infine pubblicato un lavoro sui rapporti giaciturali tra calcari e livelli riconosciuti come vulcanici nella Sicilia sud-orentale (1822) poco prima di prima di trasferirsi nell'Egitto ottomano, avendo ottenuto dall' auto-proclamato khedivè (vice-re) Mehemet Alì l'incarico di condurre ricerche rivolte allo sviluppo di attività minerarie in Sudan, dove morì nel 1826. 
1811, Memoria mineralogica sulla Valle di Fassa in Tirolo, Milano.

1814, Conchiologia fossile subapennina con osservazioni geologiche sugli Apennini, e sul suolo adiacente, 2 vols., Milano.

1817, Catalogo ragionato di una raccolta di rocce, disposto con ordine geografico, per servire alla geognosia dell' Italia, Milano.

1820, Dello stato fisico del suolo di Roma, Memoria per servire d'illustrazione alla carta geognostica di questa città, Roma.

1822, Sulle geognostiche relazioni delle rocce calcarie e vulcaniche di Val di Noto in Sicilia, Biblioteca italiana, VII (1822), pp. 53-67.

2e Scipione Breislak (1750 - 1826; Fig. 8). Nato a Roma da padre svedese, benché avesse avuto un'istruzione umanistica ben presto rivelò le sue attitudini alle ricerche nel campo della Storia Naturale mentre dal 1763, ordinato scolopo, fu insegnante in diverse scuole superiori nello Stato Pontificio (Albano, Urbino, Roma) e nel Regno di Napoli (Nola, Napoli, Tav. 1 a). In questa situazione effettuò diverse campagne di ricerca, principalmente nei distretti vulcanici laziali e campani (Tolfa, Oriolo e Latera, 1786; Campi Flegrei, 1791; Vesuvio, 1794).

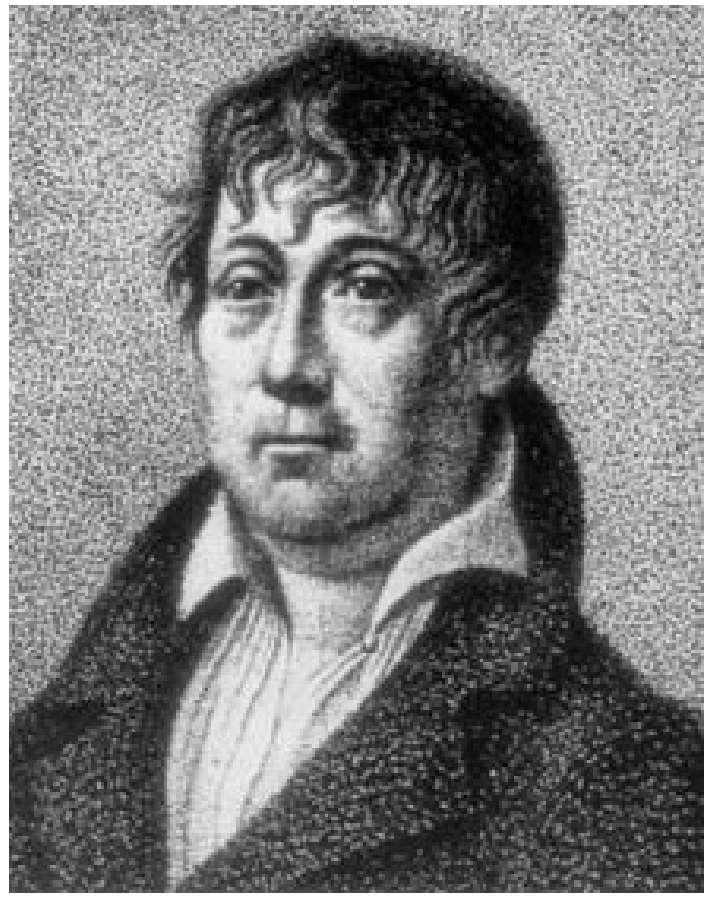

Fig. 8 - Scipione Breislak

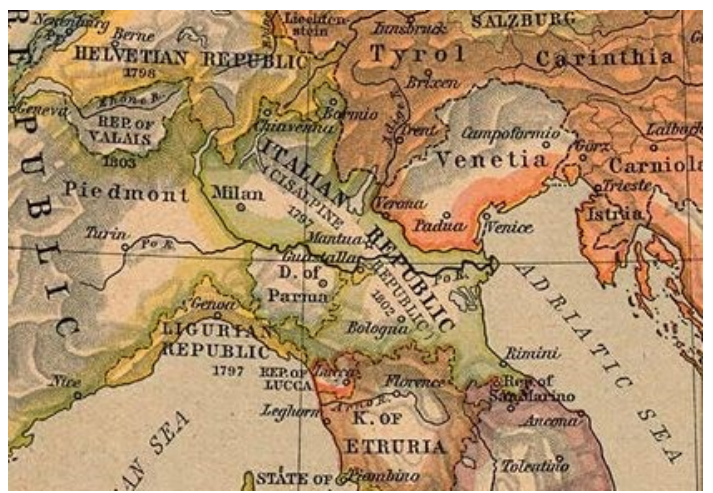

Fig. 9 - La Repubblica Italiana dal 1802 al 1804

Nel 1798 Breislak si trasferì a Roma, avendo aderito alla Repubblica Romana (Tav. 1 b) ed essendo stato coinvolto nel suo governo, ben presto caduta nel 1799. In queste circostanze andò in esilio in Francia, dove ebbe modo di incontrare alcuni dei più affermati studiosi del tempo, tra cui Cuvier, Haüy, Brongniart, e fu chiamato a leggere alcuni dei suoi saggi all'Accademia di Francia. Tornato in Italia nel 1802, a Milano fu nominato "Ispettore per la produzione di polveri da sparo e salnitro" nella Repubblica Italiana (Fig. 9; poi dal 1805 Regno d'Italia, Tav. 1 c) allora istituita, e fu in grado di proseguire le sue ricerche litologiche. Alla sconfitta di Napoleone, dopo la costituzione del Regno Lombardo-Veneto (Tav. 1 d) a seguito del Congresso di Vienna (1815), egli continuò a vivere a Milano, pubblicando alcuni lavori, anche a sostegno $(1821,1824)$ delle interpretazioni di Marzari Pencati, sulla natura magmatica di masse rocciose a contatto con calcari, contrarie all'allora ancora dominante modello nettunistico (Gennari, Rigault de la Longrais, 1972 e riferimenti). 
In ragione delle conoscenze da lui maturate sulle rocce eruttive e sui possibili processi della loro formazione, egli comprese che al suo tempo troppo poco era noto nell'ambito della fisica e della chimica per giungere ad un'interpretazione accurata dei complessi fenomeni geologici, e che si doveva raggiungere uno stretto rapporto interdisciplinare tra queste scienze e la geologia.

1786, Saggio di osservazioni mineralogiche sulla Tolfa,Oriolo e Latera, Roma.

1792, Essais minéralogiques sur la solfatare de Pouzzoles, Napoli.

1794, Memoria sull'eruzione del Vesuvio accaduta la sera de' 15 giugno 1794, in collab. con A. Winspeare, Napoli.

1798, Topografia fisica della Campania, Firenze.

1801, Voyages physiques et lithologiques dans la Campanie suivis d'un mémoire sur la constitution physique de Rome..., Paris (trad. tedesca, Leipzig 1802).

1803-1804, Notice sur la fontaine de la fumarole à la solfatare de Pouzzoles, in Journ. des mines, $\mathrm{XV}$, pp. 118-127.

1821, Sulla giacitura di alcune rocce porfiritiche e granitose osservate nel Tirolo dal Sig. Conte Marzari-Pencati. Memoria Geognostica letta all'Imperial Regio Istituto di Lombardia, Imperial Regia Stamperia, Milano.

1822, Descrizione geologica della provincia di Milano, Milano.

1824, Memorie sulle osservazioni fatte da celebri geologhi posteriormente a quelle del conte Marzari,intorno alla giacitura dei graniti del Tirolo meridionale, Milano.

1828, Osservazioni sopra i terreni compresi tra il lago Maggiore e quello di Lugano alla base meridionale delle Alpi, Memorie dell'Imperial Reale Istituto del Lombardo Veneto, vol. V, Imperial Regia Stamperia, Milano.

2f Giuseppe Marzari Pencati (1779 - 1836; Fig. 10). Nato a Vicenza (Repubblica di Venezia; Tav. 1 a) di nobile famiglia, già dalla gioventù fu principalmente interessato dallo studio della botanica, ma durante un suo soggiorno a Parigi tra il 1801 e il 1805, in pieno periodo napoleonico, il suo interesse per le scienze della Terra ebbe un forte sviluppo, dopo aver avuto l'occasione di frequentare diversi corsi e di incontrare alcuni dei più riconosciuti studiosi in queste discipline, come von Humboldt, de Jussieu, von Buch, Delamétherie e Cordier. Fin d'allora comprese che il modello nettunistico di Werner non poteva essere adottato in molte situazioni riscontrabili sul terreno (Ciancio, 2008 e riferimenti). 


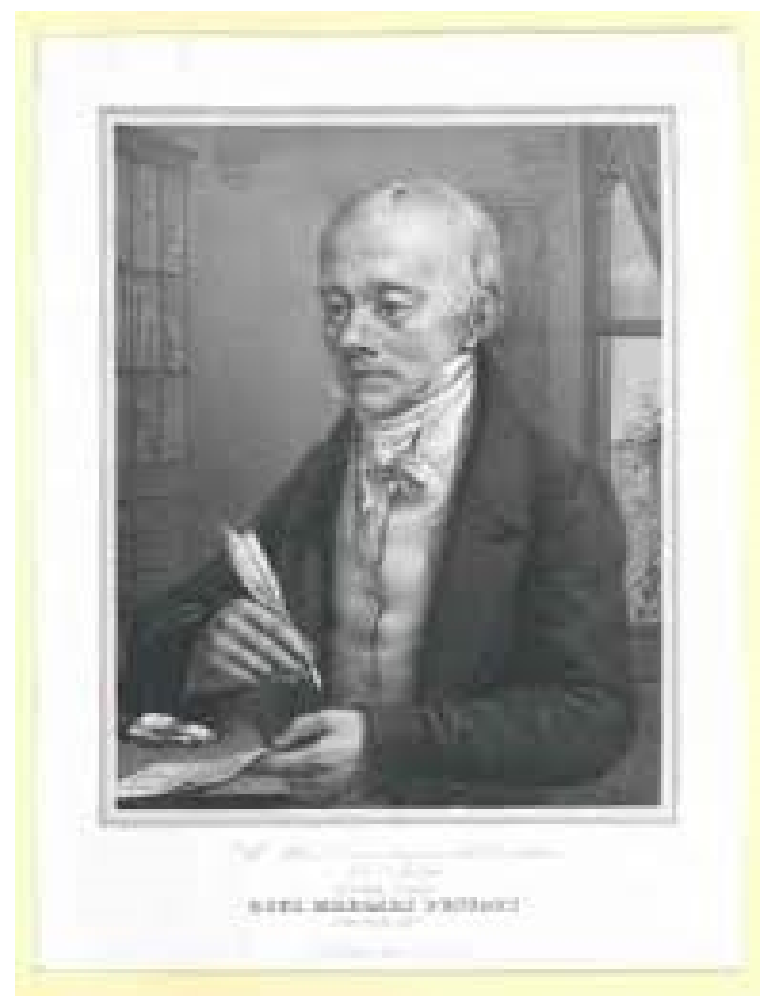

Fig. 10 - Giuseppe Marzari Pencati

Di ritorno in Italia si occupò attivamente delle attività di prospezione mineraria, che portarono ad istituire il Consiglio delle Miniere del Regno d'Italia (Tav. 1 c), nella cui regione Alpina compì numerosi viaggi, avendo avuto fin dal 1807 l'incarico di svolgere ricerche statistiche su diversi giacimenti. Anche lui, come Breislak, dopo la caduta di Napoleone nel 1814, proseguì le sue indagini e nel 1818 fu nominato Ispettore delle Miniere del Regno Lombardo Veneto (Tav. 1 d). In tale posizione trovò una convalida a quanto già intuito da Arduino e Brocchi relativamente alla presenza di "un granito in massa sovrapposto [sul fiume Avisio], in giacimento discordante, al calcare secondario" nella Valle di Fassa $(1819,1820)$, ribadita poi in diverse comunicazioni private (1822): qui trova l'evidenza di effetti di un metamorfismo termico operato dal magma granitico su calcari "secondari" (poi riconosciuti come triassici), in netto contrasto con le teorie nettuniste. In questo quadro egli riscontrò nelle stesse zone anche la presenza di livelli di transizione secondo il modello nettunista giacenti sotto porfidi (da interpretare come primari secondo Werner), interpretati come di origine magmatica.

1819, Cenni geologici e litologici sulle provincie venete e sul Tirolo, Vicenza.

1820, Notizie sopra un granito in massa sovrapposto [sul fiume Avisio], in giacimento discordante, al calcare secondario, suppl. al Nuovo Osservatore veneziano, nn. 118 e 127, pp. 1-6.

1822, Squarcio d'una lettera inedita, nella quale parlasi della giacitura del monte Cimadasta, e degli altri terreni cristallizzati terziarj posti fra il Grigno ed il Cismon. Lettera al signor Cordier. Seconda lettera al professore Cordier, Vicenza 1822.

1822, Extrait d'une lettre de Joseph Marzari Pencati à Alberto Parolini, sur le gissement de mont Cimadasta et sur les autres terrains cristallisés tertiaires situés entre le Grigno et le Cismon, in Journal de physique, XCIV (1822), pp. 316-320.

1822, Sur les granits dits tertiaires, observés en Tyrol, in Bulletin de la Société philomathique de Paris, s. 3, 1822, vol. 9, pp. 55 s.. 
1823, Porzioni della lettera geologica diretta a Giuseppe Dembsher e della sua introduzione, Vicenza 1823.

1824, Sulla sovrapposizione delle rocce di transizione di Werner al calcare secondario..., in Giorn. di fisica, chimica e storia naturale, VII (1824), 5 bimestre, pp. 377-386.

2g Carlo Gemmellaro (1787 - 1866; Fig. 11), nato a Catania (nel Regno di Sicilia, poi delle Due Sicilie, Tav. $1 \mathrm{a}, \mathrm{b}, \mathrm{c}, \mathrm{d})$ in una agiata famiglia di proprietari terrieri, fu fin da ragazzo coinvolto nell'interesse all'osservazione dell'Etna e dei suoi fenomeni eruttivi da uno zio e dal fratello maggiore, appassionati della "Montagna" (Mons - Gebel). Laureato in Medicina, avendo seguito il corso di Storia Naturale di Recupero, succeduto a Gioeni, visitò molte località di interesse geologico (essendosi arruolato come medico della marina inglese nel Mediterraneo durante le guerre Napoleoniche) nei pressi dei porti toccati dalla flotta, viaggiò nell'Europa centrale ed ebbe modo di frequentare a Londra lezioni di Humphrey Davy, incontrando anche Lyell, con cui strinse amicizia. Tornato in patria nel 1817, fu ammesso come socio dell'Accademia Gioenia al momento della sua costituzione (1824), e poi nominato professore di Storia Naturale (poi Mineralogia e Geologia, dal 1852) all'Università di Catania nel 1830 (Cristofolini, 2016 e riferimenti). Egli ebbe così modo di dare durante la sua vita uno stimolo straordinario alle ricerche geologiche e vulcanologiche in Sicilia. Per le esperienze maturate ed i rapporti stretti con studiosi di area britannica, aderì al modello che Hutton aveva proposto nel 1788.

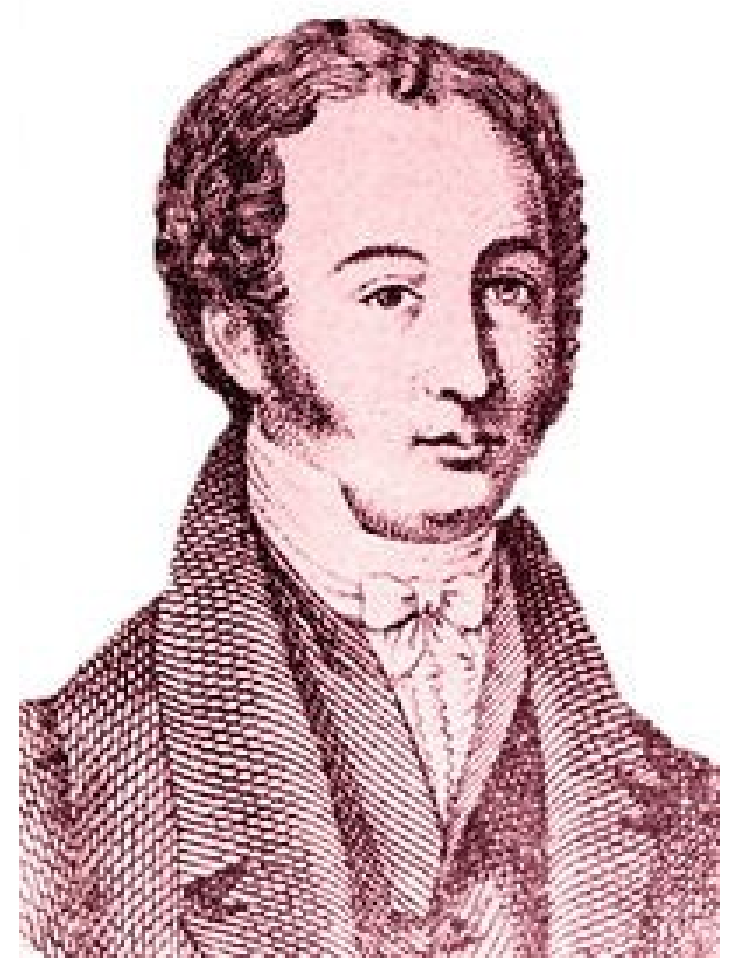

Fig. 11 - Carlo Gemellaro

Nel 1859 Gemmellaro pubblicò il suo lavoro più importante, La vulcanologia dell'Etna, sintesi dei risultati ottenuti dall'attività di ricerca di una vita sul vulcano, già esposti in decine di lavori. Fu qui tra i primi autori ad adottare il termine "vulcanologia" al fine di definire un approccio interdisciplinare allo studio dei vulcani. Nell'opera, la litologia delle successioni eruttive è assunta come criterio significativo per la distinzione di sorgenti di magma diverse alimentatrici di centri vulcanici distinti: il "feldispatico" Trifoglietto rispetto al "pirossenico" Mongibello e per classificare i relativi tipi di lava in base ad osservazioni sulle colate e su campioni, considerando anche dati chimici preliminari ottenuti (Abich, 1841) su lave e minerali, ma non considerando quelli 
pubblicati negli stessi anni da Sartorius $(1848,1853)$. Ha definito anche prodotti a fessurazione colonnare come "basalti", ritenuti come solidificati da fusi "pirogenici, non vulcanici" risaliti dalla "regione del fuoco centrale" a motivo del potere del calore, a differenza delle lave vere e proprie portate alla superficie per l'azione dell'espansione dei gas; ha inoltre trovato evidenze di effetti termici indotti da lave su materiali preesistenti e dell'alterazione (degradazione) delle lave.

Oltre che dell'Etna, si è occupato anche di vulcaniti intercalate in diversi livelli sedimentari "terziari" affioranti nella Sicilia sud-orientale (Val di Noto), interpretati come dovuti ad una migrazione verso nord delle relative sorgenti di magma $(1829,1834,1865)$. Nel campo dei prodotti sedimentari ha poi suggerito una possibile natura dei depositi di zolfo nella Sicilia centrale come dovuta all'effetto della "putrefazione" di resti organici su livelli gessosi $(1834,1835,1837)$. Ha trattato anche tematiche che oggi potrebbero essere considerate di Petrografia applicata (1838). Dopo essersi occupato in molte occasioni di arricchire le collezioni di minerali e rocce dell'Università, nel 1864, acquisì in condizioni di finanziamento critiche una collezione di rocce "da tutti i terreni della crosta del Globo" classificate secondo il sistema di von Leonhard (1723).

1829, Su i vulcani estinti di Val di Noto, Atti Acc. Gioenia Sci. Nat., 3, 211-230.

1834, Considerazioni geologiche sullo zolfo, Giorn. Gab. Lett. Acc. Gioenia, 1, 1-10.

1835, Considerazioni geologiche sullo zolfo, Atti Acc. Gioenia Sci. Nat., 10, 161-200.

1835, Sopra i vulcani estinti del Val di Noto, Mem. II, Atti Acc. Gioenia Sci. Nat., 10, 61-96.

1837, Delucidazioni sulla teoria dello zolfo, Efemer. Scient. Letter., 6, 46.

1838, Geognosia applicata all'arte del fabbricare. Delle pietre da taglio e da decorazione, Atti Acc. Gioenia Sci. Nat., 4, 49-55.

1854, Nuovi schiarimenti sulla teoria dello zolfo, Atti Acc. Gioenia Sci. Nat., s. 2, 10.

1858, La vulcanologia dell'Etna, 267 pp., Tipogr. C. Galatola, Catania, and 1859-1860, in Atti Acc. Gioenia Sci. Nat., s. 2, 14, 183-350; s. 2, 15, 27-140.

1865, Nota sui vulcani estinti del Val di Noto, Atti Acc. Gioenia Sci. Nat., s. 2, 20.

\section{Conclusioni}

Quando fu introdotto da Linneo un primo tentativo tassonomico moderno di prodotti naturali ( $S y$ stema Naturae. 1735), che includeva nel Regnum lapideum il capitolo "Rocce" (Petrae), questo schema fondato sulla suddivisione in Generi e Specie, benché inadatto alla classificazione di oggetti inanimati, fu inizialmente usato anche da diversi studiosi che operavano in questo ambito (p.e. Haüy, 1822). Dopo che già Werner (1774) aveva suddiviso le rocce in gruppi secondo criteri che tenevano conto della loro composizione e dei rapporti reciproci, dalla Mineralogia e Geologia, che a loro volta prendevano forma nella cornice della Storia Naturale, stava gradualmente sviluppandosi la Petrografia come disciplina autonoma in seguito agli importati contributi di Cordier (1816), di von Leonhard (1823) e di Brongniart (1827). Benché i nuovi criteri tassonomici introdotti fossero ancora soggetti a incongruenze ed ingenuità, si stava così compiendo un processo di gestazione millenaria, sostenuto da una forte crescita delle conoscenze scientifiche e dallo sviluppo di metodi e strumentazioni di ricerca, che avevano avuto inizio nei secoli immediatamente precedenti (Cirrincione, 2016).

Tra la fine del XVIII e la prima metà del XIX secolo alcuni italiani, tra cui noti studiosi di Storia Naturale, come L. Spallanzani, G. Gioeni, C. Gemmellaro, ed esperti in prospezione mineraria e naturalisti appassionati, come G. Arduino, G. Brocchi, S. Breislak e G. Marzari Pencati, diedero notevoli contributi alla crescita di un moderno approccio allo studio delle rocce orientato ad interpretarne correttamente l'origine ed i rapporti reciproci, in base all'attenta raccolta di dati di campagna e di osservazioni mesocopiche di campioni. All'epoca i risultati delle loro ricerche ebbero 
un'estesa circolazione e furono ampiamente apprezzati nel mondo scientifico. Complessivamente è da mettere in rilievo che tutti gli studiosi sopra citati hanno privilegiato l'interpretazione dei dati osservati direttamente all'applicazione di principi teorici: seguendo questo criterio il loro più rilevante contributo appare qui il corretto riconoscimento della natura magmatica di corpi rocciosi sia intrusivi che vulcanici inseriti in successioni sedimentarie nelle quali non avrebbero potuto trovarsi secondo il modello nettunistico suggerito da Werner a quel tempo ancora dominante.

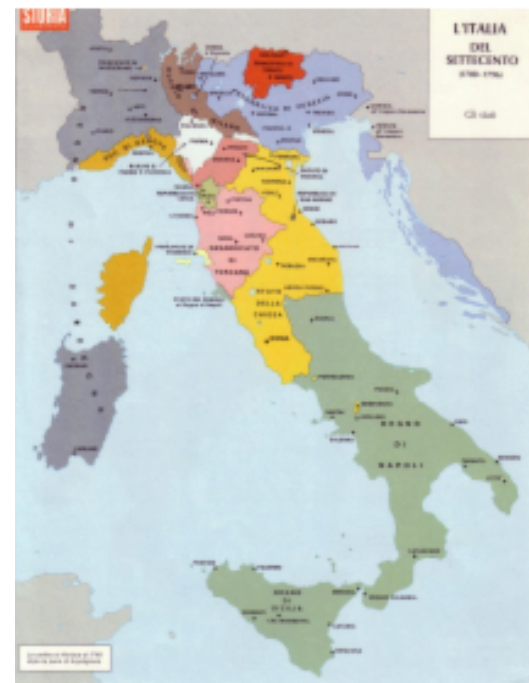

a) L'Italia prima della Rivoluzione Francese

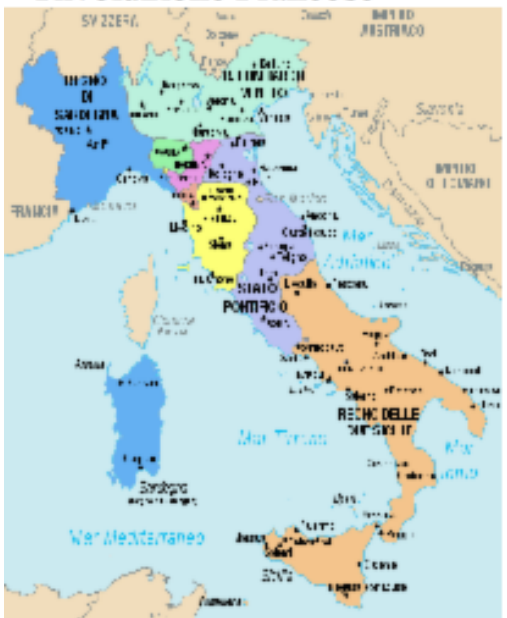

d) L'Italia dopo la Restaurazione

Tavola 1 Gli stati italiani nel periodo considerato

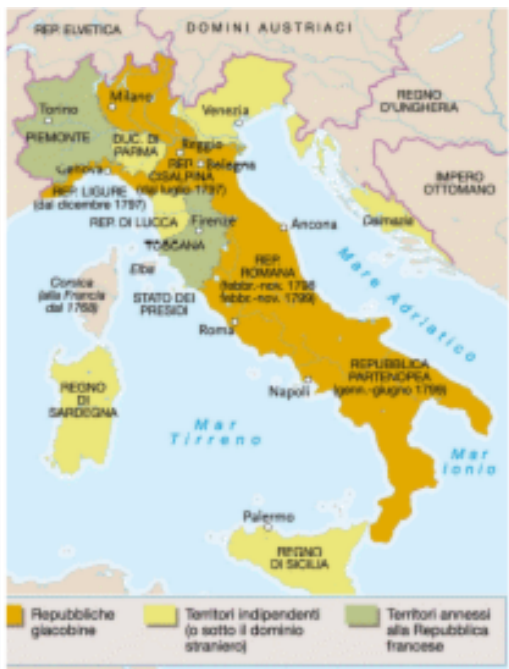

b) L'Italia nel periodo (1797-99) delle repubbliche giacobine

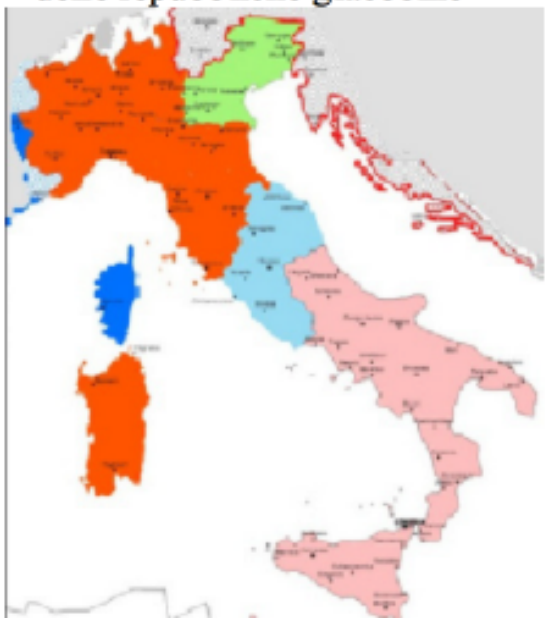

e) L'Italia nel 1859

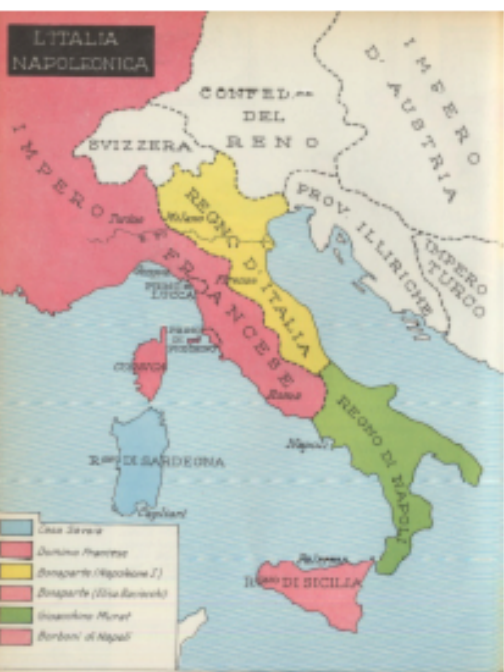

c) L'Italia durante l'impero Napoleonico

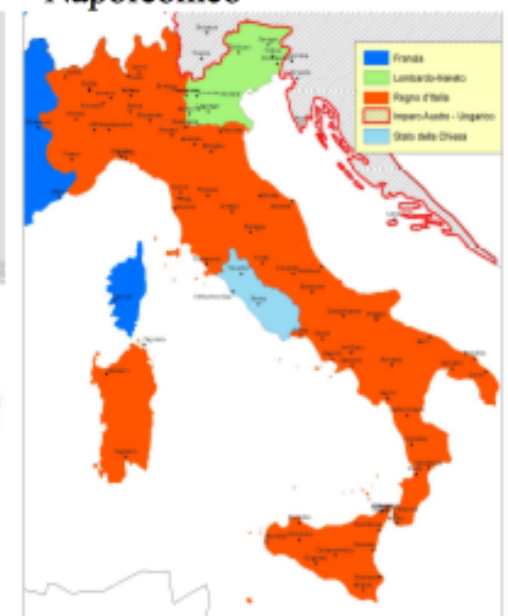

f) L'Italia dopo il 1861

\section{Riferimenti bibliografici}

[1] Abich O.W. 1841, Über die Natur und den Zusammenhang der vulkanischen Bildungen, Geol. Beobachtungen über die vulk. Erscheinungen, vol. 1, fasc.1.

[2] Brongniart A. 1827, Classification et caractères minéralogiques des roches homogènes et hétérogènes, Paris.

[3] Bruccheri G. 2001, Gioeni Giuseppe, in Dizionario Biografico degli Italiani - Volume 55, Enciclopedia Italiana Treccani, www.treccani.it. 
[4] Ciancio L. 2008, Marzari Pencati, Giuseppe, in Dizionario Biografico degli Italiani - Volume 71, Enciclopedia Italiana Treccani, www.treccani.it.

[5] Cirrincione R. 2015, La petrografia nella storia della scienza, Boll. Acc. Gioenia, 378, FP 52-79.

[6] Cordier P.L.A. 1816, Mémoire sur les substances minerales dites en masse qui entrent dans la composition des Roches Volcaniques de tous les âges, Paris, M.V. Courcier Ed.; pp. 87.

[7] Cristofolini R. 2016, From Natural History to Volcanology, Boll. Acc. Gioenia, 379, FP23 38.

[8] Gemmellaro C. 1862, Sommi capi di una Storia della Geologia sino a tutto il secolo XVIII .., Atti Acc. Gioenia, s. 2, 18, 1-40.

[9] Gennari L.G. Rigault de la Longrais G. 197, Scipione Breislak, in Dizionario Biografico degli Italiani - Volume 14, Enciclopedia Italiana Treccani, www.treccani.it.

[10] Giacomini V. 1972, Brocchi Giovanni Battista, in Dizionario Biografico degli Italiani - Vol. 14, Enciclopedia Italiana Treccani, www.treccani.it.

[11] Haüy R. J. 1784, Essai d'une Théorie sur la Structure des Crystaux, appliquée à plusieurs Genres de Substances Crystallisées.

[12] Haüy R. J. 1801, Traité de minéralogie (5 vol.).

[13] Haüy R. J. 1822, Traitè de minéralogie, II ed.,Paris, Bachelier successeur M.V. Courcier , 4 voll..

[14] Hessel J. F. Ch. 1830, Krystallometrie oder Krystallonomie und Krystallographie. Gehlers physikalisches Wörtbuch, Leipzig, pp. 1023-1360. Schwikert, Leipzig, 1830. Reprinted in Ostwald's Klassiker der Exakten Wissenschaften. Engelmann, Leipzig, 1897. 32 classi.

[15] Hutton J. 1788, Theory of the Earth, or an investigation of the laws observable in the composition, dissolution and restoration of land upon the globe, letta nel 1785.

[16] Leonhard K.C. von 1823, Charakteristik der Felsarten, vers. inglese,180 pp. Ed. Biblio Bazaar (2009).

[17] Linnaeus C. 1758, Systema Naturae per Regna Tria Naturae, secundum classes, ordines, genera, species, cum characteribus, differentiis, synonymis, locis., mprensis Laurentii Salvii,. $\mathrm{X}$ edit., Stockholm.

[18] Mazzarello P. 2013, Spallanzani Lazzaro, in Il Contributo italiano alla storia del Pensiero Scienze Enciclopedia Italiana Treccani, www.treccani.it.

[19] Romé de l'Isle JBL 1783, Cristallographie ou description des formes propres à tour les corps du règne minéral dans l'état de combinaison saline, pìerreuse ou métallique, Imprim. Monsieur, Paris.

[20] Romé de l'Isle JBL 1784, Des caractères extérieurs des minéraux, chez l'auteur.

[21] Sartorius v. Waltershausen W. 1848-1861, Atlas des Aetna, in 8 fascicoli e testo, Weimar.

[22] Sartorius v. Waltershausen W. 1853, Ueber die vulkanische Gesteine in Sicilien und Island, und ihre submarine Umbildung, Goettingen.

[23] Sorby HC 1858, On the microscopical structure of crystals indicating the origin of minerals and rocks, Quart. Journ. Geol. Soc.. 
[24] Steno N. (Stenone, Stensen) 1669, De solido intra solidum, Tipogr. Stella, Firenze.

[25] Vaccari E. 2013, Giovanni Arduino, in Il Contributo italiano alla storia del Pensiero Scienze, Enciclopedia Italiana Treccani, www.treccani.it.

[26] Werner G. 1774, Von den äusserlichen Kennzeichen der Fossilien, Lipsia, Ed. S. Lebrecht Crusius. 